\title{
Parametrization of spectra of plasma bubble induced VHF satellite scintillations and its geophysical significance
}

\author{
P. N. Vijayakumar and P. K. Pasricha \\ Radio Science Division, National Physical Laboratory, New Delhi -110012, India
}

Received: 18 March 1996 / Revised: 21 October 1996 / Accepted: 24 October 1996

\begin{abstract}
An important component of ionospheric plasma irregularity studies in the Indian low latitudes involves the study of the plasma bubbles which produce intense scintillations of the transionospheric satellite signals. Many such plasma bubble induced (PBI) scintillation events were identified while recording $244 \mathrm{MHz}$ signal from the geostationary satellite Fleetsat $\left(73^{\circ} \mathrm{E}\right)$ at Delhi $\left(28.6^{\circ} \mathrm{N}, 77.2^{\circ} \mathrm{E}\right)$ during March-April 1991. This type of scintillations represents changes in plasma processes. These scintillations are spectrally analyzed using an autoregressive (AR) scheme, which is equivalent to maximum entropy method of spectrum analysis, amenable to extracting optimum spectral content from short data lengths $(20-40 \mathrm{~s})$. Each spectrum is assigned a level of detectability using the final prediction error (FPE) derived from the optimum filter order required to resolve the spectrum. Lower detectability together with a higher order filter indicate a higher level of coherence for the plasma irregularities (discrete structures). Consistent patterns for these scintillations emerge from the present analysis as follows: (1) the initial and final phases of a scintillation patch display quasiperiodic oscillations. Their corresponding spectra show dominant (Gaussian shaped) spectral features with detectability levels of $-6 \mathrm{~dB}$ to $-12 \mathrm{~dB}$ and requiring a higher order $(>6)$ AR filter for their spectral resolution. These are most likely associated with discrete "filament-like" or "sheet-like" plasma structures that exist near the bubble walls. (2) Two main features of the scintillation spectra could be positively associated with the welldeveloped plasma bubble stage: (a) spectra displaying a power-law process with a single component spectral slope between 1.6 to 3.0. Generally such spectra are resolved with a 2 nd order filter and have a $1 \mathrm{~dB}$ to $6 \mathrm{~dB}$ of detectability. (b) Spectra displaying a double slope, indicating an inner and an outer scale regime for the power-law irregularities. These spectra are resolved with higher order filters $(>3$ but $<7$ ) and possess detectability levels of $-1 \mathrm{~dB}$ to $3 \mathrm{~dB}$. These spectra display finer spectral changes, perhaps indicative of the nature of continuously evolving plasma irregularities. As an
\end{abstract}

example, an analysis of a single scintillation patch is presented to highlight the geophysical significance of the present approach. Some important parameters used in the AR scheme of spectral analysis are given in the Appendix.

\section{Introduction}

Many investigations of ionospheric scintillation spectra have been carried out, chiefly to relate these to the scale size and amplitude of electron density fluctuations in the ionosphere. This has led to a very good understanding of ionospheric irregularities when the scattering is weak, as the problem is linear. Further, in weak scattering, diffraction effects predominate and irregularities of the scale-size of the first Fresnel zone $(\lambda z)^{1 / 2}$ are most effective in scattering the radio wave. Most of the work relating to strong scatter cases, however, are limited in scope due to the non-linear relation of the observed scintillations with the basic ionospheric irregularity parameters. Scintillations due to plasma bubbles sometimes display the effects of weak scattering in the initial stages. But, later on strong scattering effects dominate.

One significant result by Somayajulu et al. (1984) provides a direct experimental evidence for the existence of the "equatorial control" of ionospheric plasma processes observed in the Indian low latitudes especially in the pre-midnight periods of equinoxial months during high solar activity. This result is based on multistation satellite scintillation observations. These scintillations are attributed to rising plasma bubbles over the geomagnetic equator. Dabas and Reddy (1990) have systematically estimated the plasma bubble rise velocities in the Indian sector, and the values given by them are $128-416 \mathrm{~m} / \mathrm{s}$ between $450-550 \mathrm{~km}, 38-327 \mathrm{~m} / \mathrm{s}$ between $550-1140 \mathrm{~km}$ and $15-200 \mathrm{~m} / \mathrm{s}$ between 1140 $1270 \mathrm{~km}$. However, from some of our recent observations we find that the bubble rise velocities are consistently between $33-88 \mathrm{~m} / \mathrm{s}$ at $415-525 \mathrm{~km}$ over the 
equator. These low vertical rise velocities seems to suggest that there is no differential (vertical) motion involved between the bubbles and the ambient ionosphere (in the late evening). The plasma bubble induced (PBI) scintillations are restricted to very low latitudes, for low bubble rise velocities, extending only to the Hyderabad $\left(17.33^{\circ} \mathrm{N} ; 78.50^{\circ} \mathrm{E}\right)$ region. In fact, these may be manifestations of a weak Rayleigh-Taylor instability where the required conditions for the explosive growth of the instability are not met. However, there are a few instances where the explosive growth and proliferation of the instability is seen to occur on a short time scale as shown on scintillation records and ionograms. The scintillations are then seen even as far north as Delhi. These are definitely attributed to plasma bubbles with high growth rates.

Different aspects of PBI scintillations are reported by Basu et al. (1983), Franke et al. (1984), Franke and Liu (1987) and many others. Basu et al. (1983), by combining high resolution topside in situ data of electron densities and $\mathrm{VHF} / \mathrm{GHz}$ scintillations in the equatorial region, are able to make a comparison of the features of in situ spectra with unsaturated intensity scintillation spectra. Further, they report a persistent break in the slope found between $500 \mathrm{~m}$ and $1.5 \mathrm{~km}$ with the longer scale lengths over a decade showing shallow slopes on the order of -1 to -1.5 and the shorter scales over a decade showing steeper slopes of -3 to -3.5 . Franke et al. (1984) have dealt with the interpretation and modelling of quasiperiodic diffraction patterns observed in equatorial VHF scintillation due to plasma bubbles. They observe that these patterns occur at the beginning and/or at the end of intense equatorial scintillation patches of approximately $1 \mathrm{~h}$ duration. These patterns are shown to be consistent with those formed by irregularities with east-west scale sizes of a few hundred metres that are associated with the walls (edges) of equatorial plasma bubbles. Also, sometimes these patterns display an envelope modulation. The envelope modulation could be either shallow or deep depending upon the phase relationship existing between the mutually interfering scattered wave components. Trivedi (1978) and Heron (1979) have modelled some typical fading patterns by combining the effects of three Gaussian depletions with scale sizes $350 \mathrm{~m}, 250 \mathrm{~m}$ and $250 \mathrm{~m}$ with corresponding phase shifts of 54, 24 and 18 radians on a frequency of $257.55 \mathrm{MHz}$. The spacings between the irregularities are assumed to be $700 \mathrm{~m}$ and $560 \mathrm{~m}$ and they are at a height of $350 \mathrm{~km}$. The overlapping diffraction patterns from these three Gaussian lenses can be made to produce random-looking or quasiperiodic patterns of scintillations on the ground.

In another paper, Franke and Liu (1987) analyze a long-period fading component in saturated VHF amplitude scintillation observed at Ascension Island due to equatorial plasma bubbles. They show that the typically rapid fading is often associated with slow fluctuations on time scales of several minutes with peak to peak amplitudes of about $6 \mathrm{~dB}$. These are associated with scale sizes on the ground that are more than ten times the Fresnel dimension. This longwave component is often present at the beginning or end of a patch. This is shown to be compatible with that expected for a wave (radio) encountering the sharp, vertically elongated edge of a large-scale depletion in electron density. Very interesting results have been obtained by Wernik et al. (1980) while modelling radio wave scintillations caused by equatorial ionospheric bubbles. One important result of this study shows that even when the amplitude scintillations at VHF appears to be stationary, the $\mathrm{GHz}$ frequencies show outbursts with large excursions whenever the direct ray intersects a spiky ionization structure. In fact, this result validates the earlier arguments proposed by Crain et al. (1979), while using refractive scattering mechanism, to explain SHF scintillations brought about by equatorial plasma bubbles. The plume structure is presumed to play a dominant role in providing the lens action needed to explain SHF scintillations. In yet another computer simulation Vijayakumar and Tyagi (1995) have shown the strong scattering effects of spiky ionospheric structures on the GPS systems on ground. A phase screen model, used for the scattering screen at the ionospheric height, is derived from the AE-C satellite in situ data of fluctuating electron density inside a plasma bubble. Large phase jitters of GPS L1 and L2 carriers are seen on the ground. These jitters distort and decorrelate the common p-code modulation wave forms present on these two carriers. Further, the simulation shows that the cross-correlation of these distorted wave forms produce spurious correlation peaks that might completely mask or blur the wanted correlation peak due to the ionospheric group delay. This group delay is incorporated as the required ionospheric correction.

Through a myriad of results based on observation and simulation of PBI scintillations it is often impossible to comprehend the whole process in one cogent, simplified manner. Our attempt here is to present a systematized approach, to a rather complex problem of understanding PBI scintillations, through a well-proven spectral analysis technique which is useful in parametrizing the observed PBI scintillations. For this purpose a generalized autoregressive scheme of spectral analysis has been developed. It effectively deals with the problem of fixing the order of an AR process through the evaluation of multiple correlations and that of fixing the data sample length through the use of persistence in the non-random data. The present methodology is very much similar to an earlier scheme given by Ulrych and Bishop (1975). However, an important work by Kendall and Stuart (1966) on an AR process is incorporated here. Some details of the AR scheme adopted in the present analysis is given in the Appendix.

\section{Results}

Scintillation data were recorded at Delhi $\left(42^{\circ} \mathrm{N}\right.$ dip) using $244 \mathrm{MHz}$ radio signals from Fleetsat geostationary satellite during March-April 1991. Simultaneous Lband (1537 MHz) scintillation records are also available at Delhi for the same period. These L-band scintillations 
were recorded using signals from the geostationary satellite Inmarsat positioned at $63^{\circ} \mathrm{E}$ longitude over the Indian Ocean zone. The period March-April 1991 represents the start of the descending phase of the last solar-maximum. Also, Delhi happened to be the northernmost among the 19 stations which recorded the Fleetsat scintillations in a campaign mode in MarchApril 1991. A number of important scientific results based on this campaign has been reported by Chandra et al. (1993). Delhi falls outside the daytime anomaly zone. But, its sub-ionospheric point $\left(26.23^{\circ} \mathrm{N} ; 76.94^{\circ} \mathrm{E}\right)$, corresponding to $350 \mathrm{~km}$ altitude with reference to the Fleetsat $\left(73^{\circ} \mathrm{E}\right)$ ray path, lies in the vicinity of the anomaly peak. This situation is conducive for observing very intense scintillations. Indeed, these have been observed at Delhi for the said period.

At Delhi the scintillation data is recorded on a PCbased data logger at a sampling interval of about $0.1 \mathrm{~s}$. More than 300 scintillation spectra have been obtained for analysis. All these have been identified with PBI scintillations marked by the characteristic signature of rapid intensity scintillations at the start and/or at the end of these scintillations. As an example, the analysis of scintillation data of 11 March, 1991 is presented in detail.

Before proceeding further, it is worth summarizing a few (qualitative) results reported by Chandra et al. (1993) using the multistation (campaign) data of which the present data set is a part. This is intended to provide a relevant background to the present work. It is found that the scintillations generally start between 1930 and $2000 \mathrm{~h}$ IST (1400-1430 UT). The stations close to the magnetic equator show strong scintillations which last till the early morning. For the stations in the anomaly crest region and beyond, scintillations occur in patches, typically, of 15 to 90 min duration. From the occurrence pattern it is seen that scintillations occur $50 \%$ of the time in the equatorial zone, $30 \%$ of the time in the anomaly crest region and only $10 \%$ of the time at Delhi, being the northernmost station. One significant observation made is that if the scintillations are seen already broken up into patches at the geomagnetic equator itself, then the scintillations are less likely to be seen at the crest latitudes and northward.

Next, some qualitative aspects of PBI scintillations recorded on 11 March, 1991 are described. In Fig. 1 the three distinct features corresponding to the initial phase of the first scintillation patch recorded at Delhi on 11 March, 1991 is shown. A very good estimate of the west to east drift velocity of this patch has been made by noting the exact time delay (=730 s) in the occurrence of Fleetsat $244 \mathrm{MHz}$ scintillations with respect to L-band scintillations recorded from Inmarsat $\left(63^{\circ} \mathrm{E}\right)$. The subionospheric points, relating to $\mathrm{F}$ region height, of these two satellites are separated by a distance of $82 \mathrm{~km}$ which would result in a west-east velocity, $U$, of about $112 \mathrm{~m} / \mathrm{s}$. Moreover, if the height $(z)$ of the irregularities is assumed to be at $350 \mathrm{~km}$, then the Fresnel break frequency is $0.171 \mathrm{~Hz}$ from Eq. (1). This is further confirmed through the spectral analysis of the typical Fresnel type fading, seen in stage 1 of Fig. 1, often as-

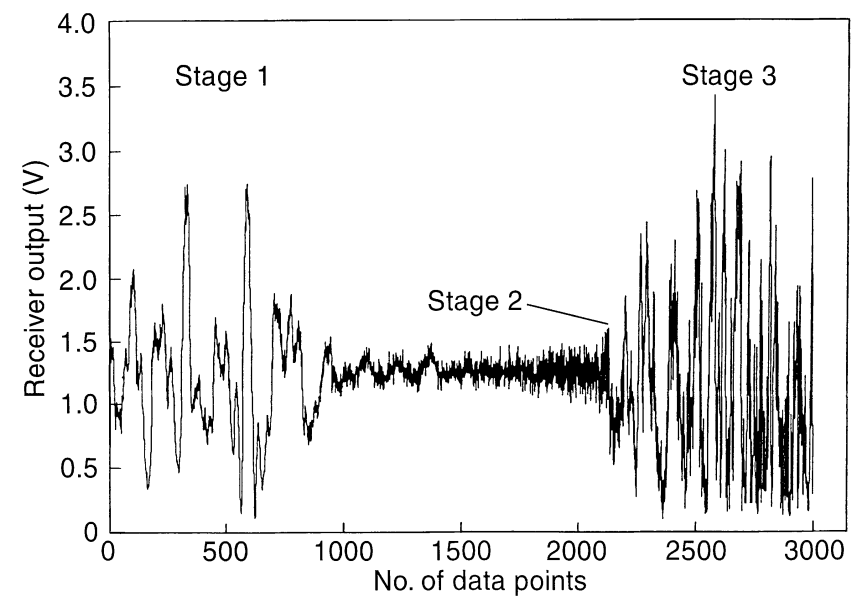

Fig. 1. Three different scintillation patterns are shown here. Stage 1 is associated with a discrete irregularity structure, stage 2 is a weak manifestation of quasiperiodic scintillation and stage 3 is the pattern of strong scintillations due to the well-developed plasma bubble. This is the very first patch of scintillation recorded on 11 March, 1991

sociated with a well-defined Gaussian irregularity structure. In Fig. 2, the power spectrum, obtained through the AR scheme, corresponding to stage 1 (of Fig. 1) data is shown. The theoretically derived Fresnel break frequency given by,

$f_{B}=U /(\lambda z)^{1 / 2}$

(where the values of relevant parameters on RHS are taken from Table 2) is seen to match well with the break frequency, $f_{B}$, marked in Fig. 2, which corresponds to $0.171 \mathrm{~Hz}(171 \mathrm{mHz})$. This is a useful reference parameter for the spectral estimates that are obtained here. The spectrum in Fig. 2 is resolved with a 9th order AR filter and possesses a detectability level of $-12 \mathrm{~dB}$. This is a higher order AR process representing a discrete structure.

Next, the power spectra of PBI scintillations at various stages of plasma bubble evolution need to be estimated. From these one can estimate the irregularity

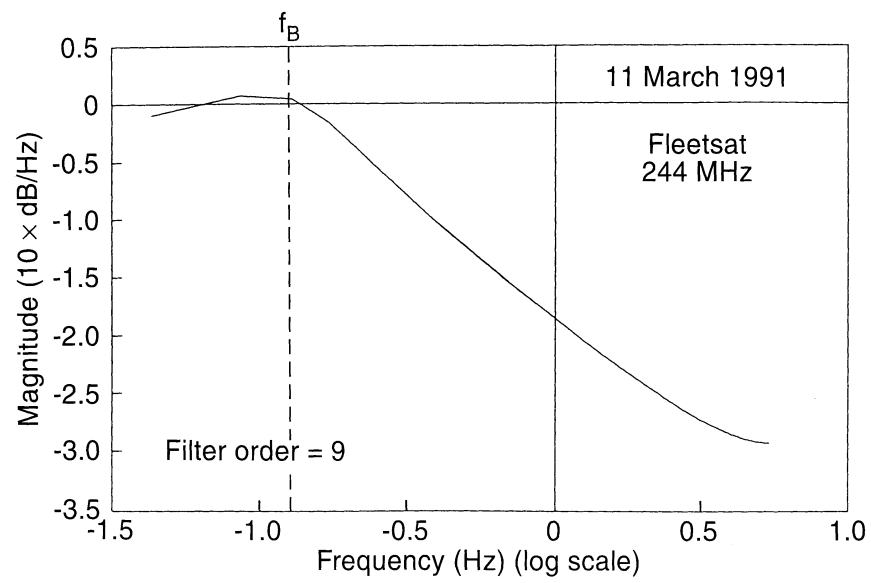

Fig. 2. The power spectrum of stage 1 scintillation. The break frequency, $f_{B}$, is clearly marked and corresponds to the theoretically calculated Fresnel frequency of $0.171 \mathrm{~Hz}$ 
scale-sizes involved in the production of the PBI scintillations. In the present analysis the spectral window has been limited to observing the spectral features between " $f_{B}$ "--Fresnel break-frequency $(0.17 \mathrm{~Hz})$, and " $f_{N}$ "-Nyquist frequency $(5.5 \mathrm{~Hz})$. This converts to the scale sizes of $656-20 \mathrm{~m}$ due to the drift velocity $(112 \mathrm{~m} / \mathrm{s})$. Further, the (spectral) resolution bandwidth used in the processing corresponds to a spatial resolution of $20 \mathrm{~m}$ for the irregularity spectrum.

In the early part of stage 2, Fig. 1, no scintillation is present. However, scintillation build-up is seen at a later part of stage 2, which is distinctly marked on Fig. 1. This portion of stage 2 is magnified in Fig. 3a. Figure 3a shows a well-known scintillation signature, often associated with diffraction of radio waves at the edge of a plasma bubble. The radio waves display fast signal amplitude fluctuations with typical (quasi) periodicities of $0.2-0.3 \mathrm{~s}$. These fast fluctuations may well be (Franke and Liu, 1984) due to the presence of discrete plasma structures of typical dimensions of a few hundred metres existing near the bubble walls. This is further established by their model simulation by Liu and Franke (1986). As previously mentioned, at times, these fast fluctuations are also seen to be contained within an envelope with much slower fading of the order of 10 to $15 \mathrm{~s}$, clearly indicating the presence of a group of waves (3 waves). In

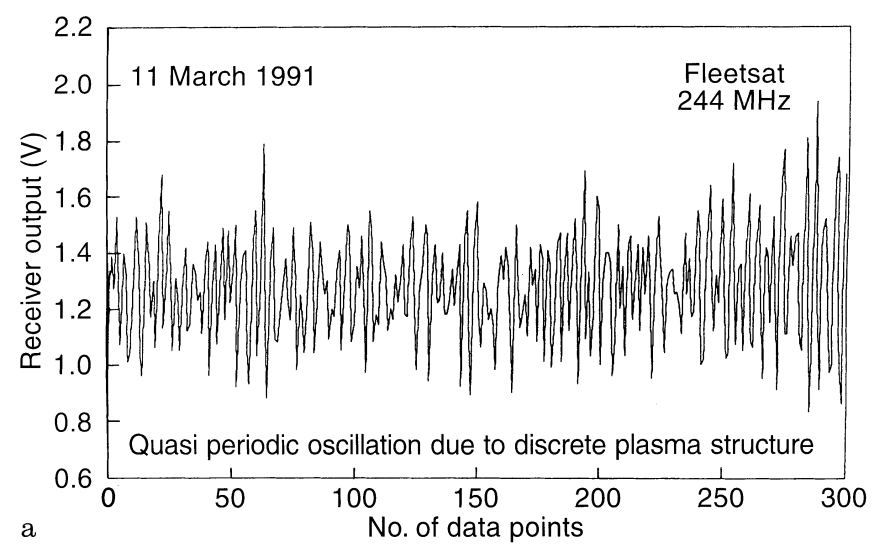

a

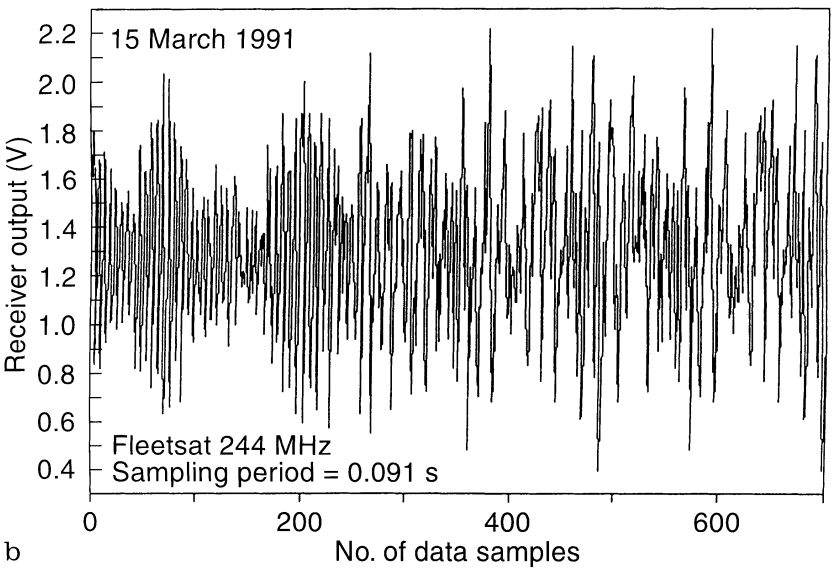

Fig. 3. a Well-resolved quasiperiodic patterns recorded on 11 March, 1991 are seen here. The fading time is found to be about $0.3 \mathrm{~s} ; \mathbf{b}$ welldeveloped quasiperiodic pattern recorded on 15 March, 1991 is shown here. Modulation envelopes are clearly seen
Fig. $3 b$ is shown the example of a typical envelope, recorded on 15 March, 1991. However, there is no such envelope seen in Fig. 3a. This is explained as either due to the absence of any discrete structure near the bubble wall or the existence of a very small scale-size of irregularity (a few tens of metres) existing at the bubble walls where thickness $L$ of the irregularity layer is about one kilometer. Figure 4 shows the typical power spectrum corresponding to fast scintillations shown in Fig. 3a. This spectrum is plotted on a linear scale, to highlight the deterministic nature of the spectrum which is Gaussian shaped with a narrow line width. This spectrum is resolved by a 9th order AR-filter with detectability exceeding $-6 \mathrm{~dB}$. The corresponding S4index (normalized variance of signal intensity) is evaluated as 0.12 for this portion of the record. By taking the spatial conversion due to the E-W motion $(=112 \mathrm{~m} / \mathrm{s})$, the peak of this spectrum in Fig. 4 would correspond to a scale size of $33 \mathrm{~m}$. This is clearly an order of magnitude less compared to the scale sizes of irregularities giving rise to envelope modulation of the quasiperiodic fluctuations as shown by Franke et al. (1984). Also, the time extent of stage 2 as noted from the data is approximately $114 \mathrm{~s}$. This produces a ground-extent of $12.78 \mathrm{~km}$ for the pattern, again assuming the drift velocity to be $112 \mathrm{~m} / \mathrm{s}$ for the pattern on ground. From this value the gradient, $\phi / d(\operatorname{rads} / \mathrm{m})$ can be obtained at the bubble edge by substituting appropriately into the Titheridge (1971) formula for one half of the ground extent of the pattern given by,

$D=5 \times 10^{7} h \times(\phi / d) / f$.

This is obtained from a simple ray optics model, where $D(\mathrm{~m})$ is half the extent of pattern on ground, $h(\mathrm{~m})$ is the irregularity height in the ionosphere, $\phi$ is the phase angle change (rads), $f$ is the radio frequency $(\mathrm{Hz})$ and $d$ (m) is the scale size. While dealing with the edge of a bubble in the present case, the ratio $\phi / d$ turns out to be equal to 0.089 rads $\mathrm{m}^{-1}$. This would correspond to a

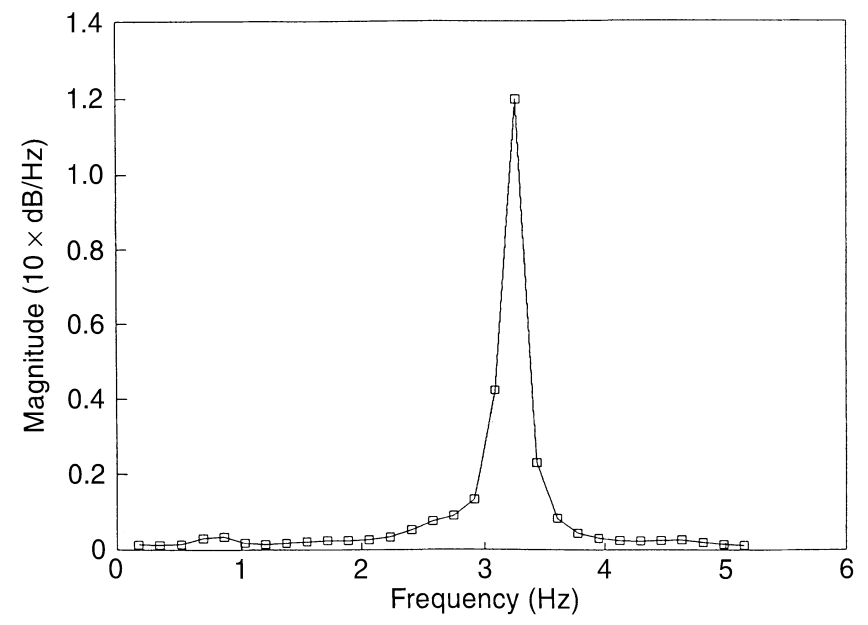

Fig. 4. The typical power spectrum obtained for stage 2 quasiperiodic scintillation. A dominant spectral peak is seen at $\approx 3 \mathrm{~Hz}$ corresponding to a spatial conversion of $\approx 33 \mathrm{~m}$ due to the drift velocity of $112 \mathrm{~m} / \mathrm{s}$ 
peak value of $\phi=3$ rads, if we assume an irregularity of scale size of $33 \mathrm{~m}$. This is what is expected for a typical "filament-like" irregularity at the eastern edge of the bubble inclining westward (depletion growing in magnitude as one proceeds westwards along the wall) and a number of such discrete structures contributing to the observed quasiperiodic fluctuations. This has been verified using simulations similar to those performed by Wernik et al. (1980). The results are found to be in agreement with the present observations.

The portion of the record marked stage 3 (in Fig. 1) is due to the expanding stage of the plasma bubble. The abrupt change in the scintillation pattern is very apparent. In Fig. 5 a typical scintillation pattern due to a well-developed bubble is seen. Figure 6 shows the pattern (reverse of pattern in Fig. 1) occurring at the end of the PBI scintillation patch. The total duration of the intense scintillations marked stage 3 is $22 \mathrm{~min}$. The start and the end of this intense scintillation patch are clearly marked in Figs. 1 and 6 respectively. During the entire

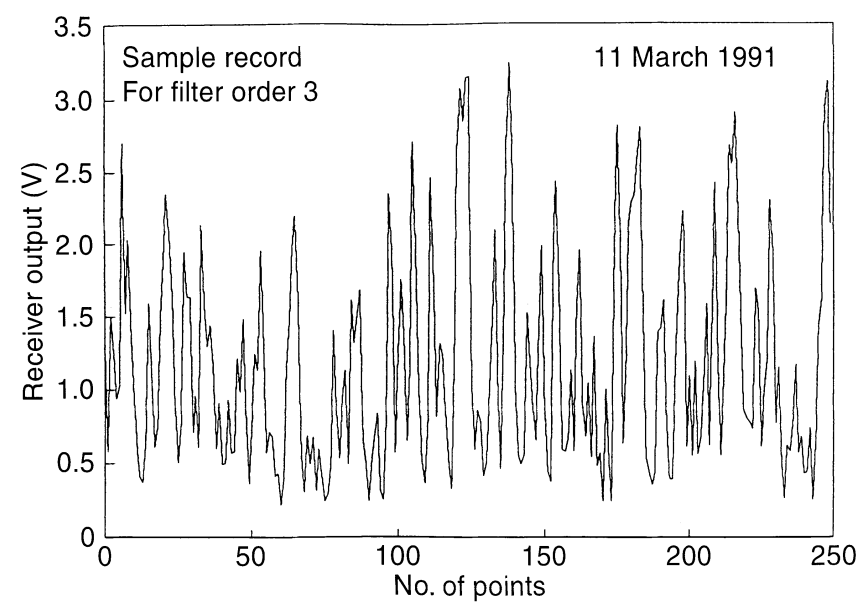

Fig. 5. A well-resolved strong scintillation pattern belonging to stage 3 representing the well-developed stage of a plasma bubble

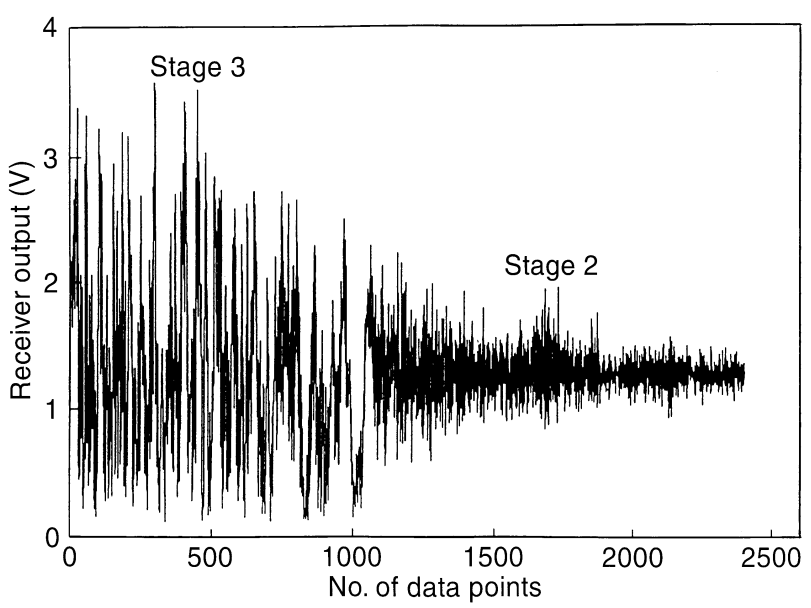

Fig. 6. Shows the weakening of the plasma bubble induced scintillation, where, there is a reversal of the trend, namely, strong stage 3 scintillations are followed by less intense quasiperiodic stage 2 scintillations. This is reverse of the trend depicted in Fig. 1 period of stage 3 scintillations the value of S4 index has remained greater than 0.5 , with the highest value being 0.9 . The end of the scintillation patch is marked by a brief break in scintillation activity, thereafter, a fresh scintillation activity is seen to begin and the whole sequence of events (illustrated already) is repeated. Again, attributing the observed time duration of the patch to the west-east drift $(112 \mathrm{~m} / \mathrm{s})$, the east-west extent of the patch (bubble) is $147 \mathrm{~km}$.

Some results of AR spectral estimates of strong scintillations, brought about by the (explosive) stage 3 of the plasma bubble evolution, are presented. A large number of spectral estimates belong to the category of strong scintillations compared to a much smaller number representing the (weak) scenario at the start or end of a PBI scintillation patch. Amongst these about 70\% of the spectra show typical power-law characteristic of flat power spectral density (psd) extending up to 2 to 3 $\mathrm{Hz}$ in frequency, beyond which the roll-off is smooth up to Nyquist frequency $(5.5 \mathrm{~Hz})$. The roll-off is characterized by a single slope $(n)$ which is seen to lie between 3.0 and 1.6. Figure 7 shows a set of power-law spectra associated with 1st-patch of scintillations of 11 March, 1991. The S4-indices is found to be between 0.5-0.8. These are definitely in the strong scatter regime. In Table 2 the important parameters resulting from the spectrum analysis of the entire data of 11 March, 1991 are shown.

Figure $8 \mathrm{a}, \mathrm{b}$ shows the finer variations in the spectral patterns with double slope features, and requiring 4th, and 5th order of AR-filters for their resolution. The range of values of these two slopes $n 1$ and $n 2$ are $0.5-1.9$ and 1.5-3.0, respectively. Typically, values of $n 1$ and $n 2$ are 1.1 and 2.6 respectively. It is found from the break of scale that the inner scale lies between $200 \mathrm{~m}$ and $50 \mathrm{~m}$. This is much smaller compared to $1.5 \mathrm{~km}-500 \mathrm{~m}$ that Basu et al. (1983) report using in situ electron density measurements. This aspect is considered in a later discussion.

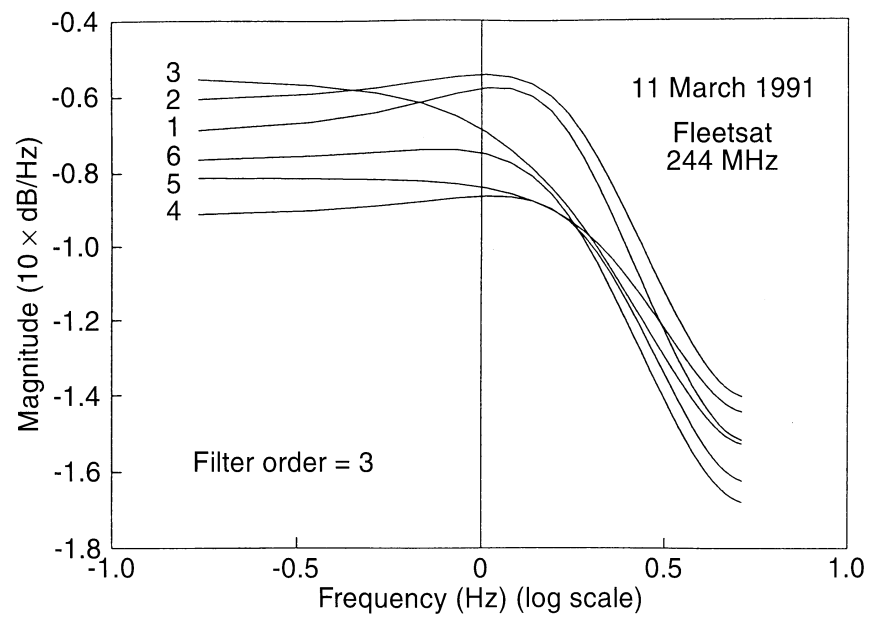

Fig. 7. Typical power law spectra represented by a flat power spectral density up to $\approx 3.3 \mathrm{~Hz}$ and the higher frequency roll-off giving a single component spectral slope. The spectra are numbered $1-6$ representing the time sequence of their occurrence 

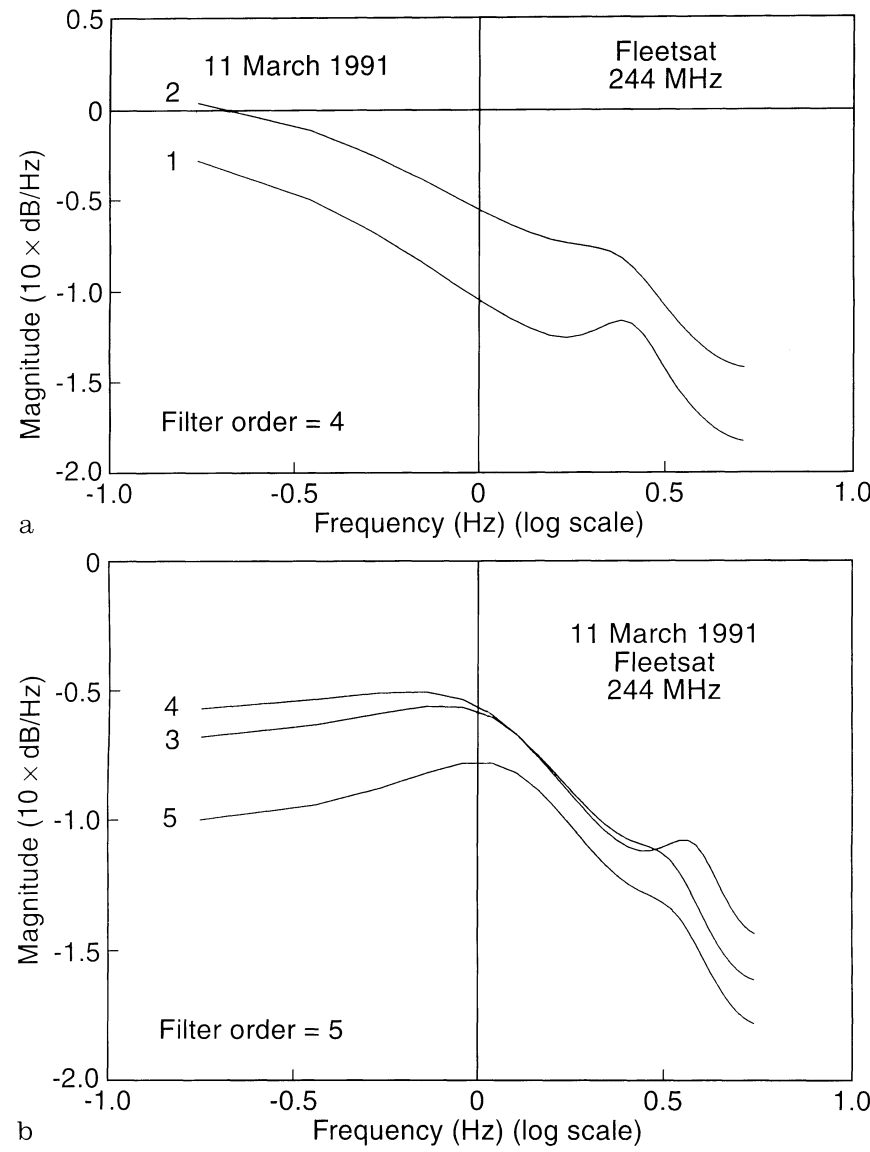

Fig. 8a,b. The power spectra of scintillations represented by higher order AR processes are shown in $\mathbf{a}$ and $\mathbf{b}$. These are resolved by 4 th and 5 th order filters respectively. Note the dual slope nature displayed by all these spectra and the finer variations in their shapes. Again the numbers represent the time sequence of occurrence of these spectra

In Fig. 9 the autocorrelation functions (ACF) are shown for the three typical cases (a) ACF associated with quasiperiodic scintillation, displaying typical narrow band noise characteristic, (b) ACF of a power-law process with a single spectral slope, displaying characteristic of wide-band noise, and (c) ACF associated with scintillations with a typical double slope spectrum; typical of a non-random process.

The most important results of the present analysis are tabulated in a very concise manner in Tables 1 and 2 . While in Table 1 the numerical values of important physical parameters are mentioned, in Table 2 a

Table 1. Numerical values of some physical parameters

\footnotetext{
Satellite radio wave frequency, $f=244 \mathrm{MHz}$

Radio wavelength, $\lambda \approx 1.23 \mathrm{~m}$

Assumed ionospheric height, $z=350 \mathrm{~km}$

First Fresnel zone for a ground observer, $(\lambda z)^{1 / 2}=676 \mathrm{~m}$

Experimentally noted irregularity drift speed, $U=112 \mathrm{~m} / \mathrm{s}$

Corresponding Fresnel break frequency, $f_{B}=0.171 \mathrm{~Hz}$

Data sampling time, $\delta t=0.091 \mathrm{~s}$

Time extent of scintillation patch due to fully developed

plasma bubble $=22 \mathrm{~min}$

One dimensional spatial extent of the plasma bubble $=147 \mathrm{~km}$
}

(consistent) set of the parameters derived from the AR scheme of spectrum analysis is shown. There seems to be a good one to one correspondance between the physical process of the plasma bubble evolution, as seen through scintillations, and its parametrization through the AR process.

In Fig. 10, the normalized final prediction error (FPE) (derived from the algorithm of Ulrych and Bishop, 1975) and the incoherent averaging (computed from method given in the Appendix) are plotted. The significance of this is that the scintillations belonging to the strong scatter regime (S4 0.5-0.8), are clearly seen to fall into two categories, when treated as an AR process. One is the well-known power-law (red noise) process, so abundantly mentioned in the literature. The other is the less known (less explained) higher order process. While the former is seen to approach an (ideal) white noise process linearly, the latter is associated with more scattering of its representative points. This is because these points represent situations of varying degrees of (intrinsic) coherence unlike the power-law process. Thus the power-law process is more amenable to incoherent averaging. This seems to suggest that the method of applying a uniform averaging may not always be correct if the process dealt with is of a higher order. Also, since the FPE is seen to be substantially lower in the case of higher order process, the process is seen to possess, inherently, a longer characteristic time scale $\left(T_{o}\right)$. The foregoing arguments are equally valid for understanding the physical attributes of the parameters shown in Table 2.

\section{Discussion}

A set of semi-quantitative results are obtained from AR spectral analysis of satellite scintillation data corresponding to March-April 1991. A distinctly consistent pattern emerges from this spectral analysis of those scintillations which are primarily induced by plasma bubbles.

Due to lack of satellite total electron content (TEC) data, and since no ionosonde was operative in the vicinity at the time of recording the scintillations, very little is known of the nature of the background ionosphere. Under the circumstances, we have tried to supplement by using some results of past satellite (ETS-II $\left.\left(130^{\circ} \mathrm{E}\right), 136 \mathrm{MHz}\right) \mathrm{TEC}$ and scintillation measurements made during the high solar activity period of 1979-1980. This is reasonable, since, the scintillation data analyzed here belongs to a sufficiently high solar activity period. Further, when we consider the TEC data for the equinox periods at Delhi, it shows a secondary peak in the late evening hours. Garg et al. (1983), analyzing the two months (January-February 1980) ionospheric electron content (IEC) data from a network of stations located within $77-79^{\circ} \mathrm{E}$ meridian and covering a latitude belt of $3-19^{\circ} \mathrm{N}$, show that the Delhi IEC enhancement is at the expense of the rate of IEC decay at a low-latitude station like Bangalore $\left(77.6^{\circ} \mathrm{E}, 3.2^{\circ} \mathrm{N}\right.$ geometrical latitude). Also, if the IEC decays gradually then there is no likelihood of 


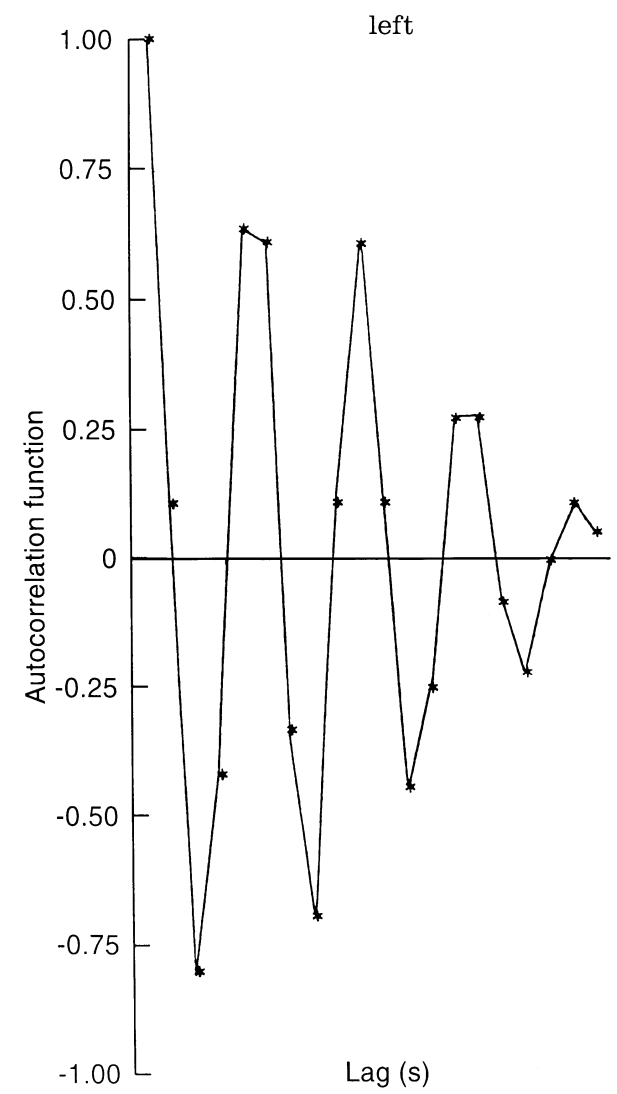

Fig. 9. The autocorrelation functions for 20-lags are plotted for three typical cases, i.e. left quasiperiodic scintillations, middle scintillations displaying power law spectra with single slope and right scintillations displaying dual slope in their power law spectra. Note that left panel

observing the secondary IEC enhancement at higher latitudes. The typical noon time peak values of TEC range between $80-120$ units (1 TEC unit $=10^{16} \mathrm{el} \mathrm{m}^{-2}$ ) and the post sunset secondary maxima occur between 2000-2230 $\mathrm{h}$ IST with the peak values reaching the noon

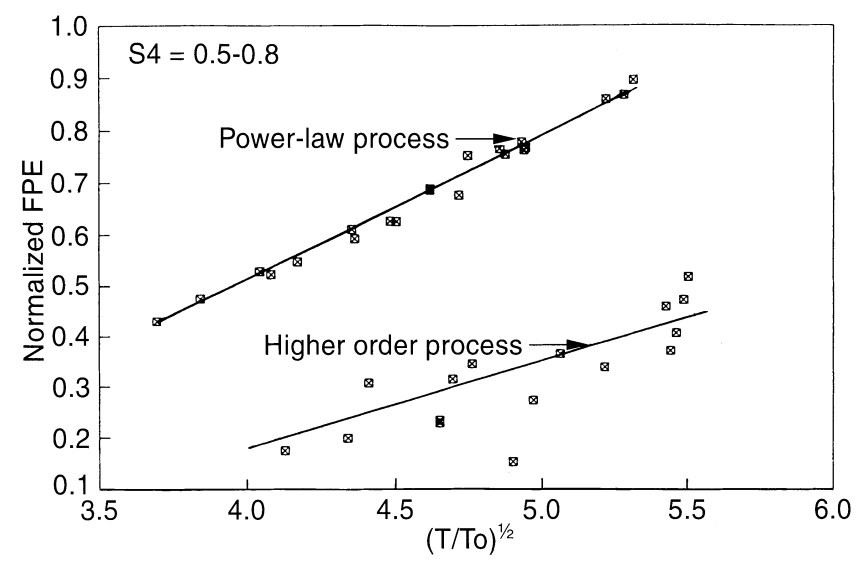

Fig. 10. This is a plot of the normalized final prediction error (obtained from AR algorithm based on Akaike criterion) versus the smoothing parameter $\left(T / T_{0}\right)^{1 / 2}$. The linear trend displayed by the power-law process indicates that the lower order processes are more amenable to smoothing than the higher order process due to the latter possessing in intrinsic coherence

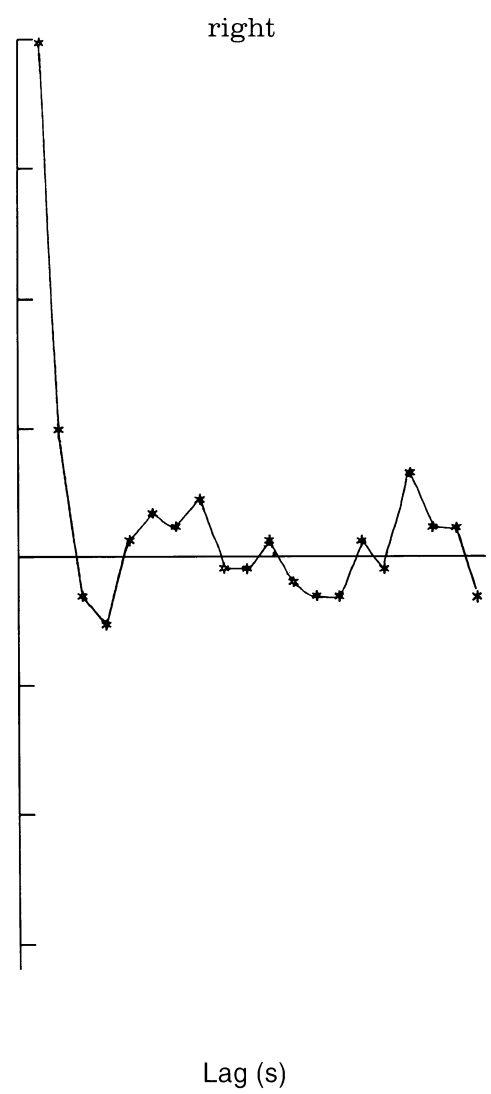

corresponds to a typical narrow-band noise-like process, middle corresponds to a wide band noise process, while right is associated with non-random process. Each lag corresponds to the sampling time, $\delta t=0.091 \mathrm{~s}$

time values. However, the most suitable secondary TEC peak values are between $80-90 \%$ of the main peak for observing large TEC bite outs (depletions) because plasma bubbles give rise to intense scintillations often accompanied by Faraday polarization fluctuations. So, a secondary peak value of 100 TEC units and $f_{0} \mathrm{~F}_{2}$ of 15 $\mathrm{MHz}$ are assumed to represent the background ionospheric parameters. This is close to that indicated by Anderson and Klobuchar (1983) while modelling TEC over Ascension Island.

It is reasonable to presume that the background ionosphere remains mostly undisturbed just prior to the plasma bubble expansion stage. Hence, weak irregularities may appear at this stage, which might have got shorn off the edge of the bubble earlier. This situation is very well seen from the simulations performed by Chaturvedi and Ossakow (1977) and later by Zalesak et al. (1982) while dealing with the explosive (nonlinear) growth of gravity driven Rayleigh-Taylor instability, subsequently giving rise to the well-developed stage of a plasma bubble and associated irregularities. At this stage, a hierarchy of plasma instabilities set in and can generate fluctuating electric fields and plasma densities on length scales between $10 \mathrm{~cm}$ to $100 \mathrm{~km}$ and more, and on time scales of ms-mins. So, it is justified, in the present context to suppose that the quiescent conditions of the ionosphere must have existed during the stages 1 
Table 2. Parameters from AR scheme of spectral analysis

\begin{tabular}{|c|c|c|c|c|c|c|}
\hline Physical attribute & Spectral character & $\begin{array}{l}\text { Total number } \\
\text { of spectra }\end{array}$ & $\begin{array}{l}\text { Characteristic } \\
\text { time scale }\left(T_{0}\right)\end{array}$ & Slope(s) & Detectability & $\begin{array}{l}\text { S4-Index/ } \\
\text { AR-order }\end{array}$ \\
\hline $\begin{array}{l}\text { Discrete, isolated } \\
\text { structure }\end{array}$ & Gaussian & 14 & $1-2.5 \mathrm{~s}$ & - & $>-12 \mathrm{~dB}$ & $\begin{array}{c}0.15-0.35 \\
8,9\end{array}$ \\
\hline Quasiperiodic & $\begin{array}{l}\text { Dominant } \\
\text { spectral peak } \\
\text { (Quassian shaped) }\end{array}$ & 27 & $0.1-0.3 \mathrm{~s}$ & - & $>-6 \mathrm{~dB}$ & $\begin{array}{c}0.15-0.4 \\
6-8\end{array}$ \\
\hline Strong scatter & Power-law (red-noise) & 190 & $0.2-0.4 \mathrm{~s}$ & $1.6-3.0$ & $1-6 \mathrm{~dB}$ & $\begin{array}{l}0.5-0.8 \\
2,3\end{array}$ \\
\hline $\begin{array}{l}\text { Inner/outer } \\
\text { scale of power-law } \\
\text { irregularity }\end{array}$ & Double slope & 78 & & $\begin{array}{l}\text { (n1) } 0.5-1.9 \\
\text { (n2) } 1.5-3.0\end{array}$ & $-3 \mathrm{~dB}-1 \mathrm{~dB}$ & $\begin{array}{c}0.6-0.8 \\
4,5\end{array}$ \\
\hline
\end{tabular}

Tabulated above are the various parameters of significance derived from the autoregressive scheme of spectral analysis of plasma bubble induced scintillations recorded at Delhi on 11 March, 1991. The power-law process with single spectral slope can be represented as a third order AR-process. Higher orders define a degree of coherence for a given process. They are also associated with spectra of discrete plasma structures

and 2 depicted in Fig. 1 and the same is substantially disturbed to give rise to the observed intense scintillations (stage 3). Hence, we are dealing with a highly turbulent ionosphere in the well-developed stage of the plasma bubble and the irregularity layer thickness might have extended in excess of $100 \mathrm{~km}$. Also, because, very short time fluctuations are involved, it is appropriate to analyze short (scintillation) data lengths for (spectrally) appreciating the subtle changes in the data and to capture the essence of the fleeting plasma processes. Referring to Table 2, it is found that high detectability levels are associated with the spectra (flat) of strong scintillations and hence, geophysically, the plasma turbulence tends to be a white-noise process. From the corresponding scintillation data large defocusing effects are noted. These can be brought about only by irregularities that have electron density enhancements in excess of $10-50 \%$ of the background and thus acting like diverging lenses. These possess scales much larger than the Fresnel scale on the ground indicative of large density gradients at ionospheric heights. On the other hand, spectra with double slope features possess a slightly lower detectability level and are indicative of distinct inner and outer scale regimes of the irregularities. There are two types of these dual slope spectra. For the first type the outer scale is close to the Fresnel scale (as seen from the spectral roll-off) and the break of slope occurs at around $200-300 \mathrm{~m}$. The second type possesses an outer scale much smaller than the Fresnel scale $(<100 \mathrm{~m})$, because irregularities much larger than the Fresnel scale are contributing to the overall scattering and hence the psd at shorter scales are also enhanced. The inner scale is also correspondingly reduced to a few tens of metres. This entire scenario has but a very limited correspondence with the actual irregularities in the ionosphere as the prevailing situation is one of high non-linearity. However, phase screens employed in simulations can be modelled to give scintillation patterns whose spectra can be made to resemble the spectra obtained here. The spectral changes can be comprehended by changing the background ionosphere (with a height-dependent weighting function) in which the phase screen features are continually being varied in a nonlin- ear fashion, in much the same way, as done by Wernik et al. (1980). As already mentioned, a higher order filter together with lower spectral detectability is seen to be associated with a higher intrinsic coherence for the AR process. It can be, generally, inferred that scintillations possessing such an intrinsic coherence must have been produced by plasma irregularities whose spectral components must have possessed a degree of phase coherence at the ionospheric heights. This is precisely the inference drawn by Wernik et al. (1980) while explaining non-stationary features of $\mathrm{GHz}$ scintillations seen in their simulations. This is clearly seen as due to the presence of spiky plasma density features from which the model phase screen has been derived. Further, in the same simulation Wernik et al. (1980) show that if the phase coherence is randomized, then the nonstationary $\mathrm{GHz}$ scintillation features, almost vanish, but the S4 index is not altered greatly. So, from the present analysis it is possible to infer intuitively, that the spectra associated with lower detectability and represented by higher filter order may provide a clue to the existence of spiky ionospheric features and thus having ramifications for the $\mathrm{GHz}$ scintillations and satellite communications at these frequencies. So statistics of occurrence of such spectral features obtained with VHF scintillation data may provide a better input for modelling scintillation effects on satellite $\mathrm{GHz}$ frequencies.

Another important parameter is the estimation of the extent of the plasma bubble. For the example considered, it is evaluated as $147 \mathrm{~km}$. This deduction is based purely on the west-east drift velocity and the temporal extension of the scintillation patch. This is highly simplistic, because the plasma bubble is expanding while it is drifting, and very little is known regarding the rate of expansion. This argument suggests that the value obtained for the east-west extent $(147 \mathrm{~km})$ may be due to the combined effects of drift and expansion of the plasma bubble. There is yet another important consequence of this "plasma bubble expansion". This would, virtually, enhance the "overall" drift speed on ground such that the (scintillation) spectral break frequencies would also get shifted to higher (temporal) frequencies. This would mean that if a satellite probe registers a 
break in slope in the spectrum of in situ plasma density variations in $1.5 \mathrm{~km}-500 \mathrm{~m}$ range, the same would be correspondingly shifted to higher temporal frequencies depending upon the "overall" speed with which these density variations are drifting with respect to a ground observer. Of course, a fundamental assumption is that density variations are giving rise to scintillations on the ground whose spectra are being considered. These arguments may hold good in the case of those spectra, that correspond to unsaturated scintillations $(\mathrm{S} 4<1)$, but whose break frequency is seen to be shifted towards much higher temporal frequencies, as in the case of saturated $(\mathrm{S} 4=1)$ scintillations.

\section{Conclusion}

It is possible to deduce some important ionospheric parameters from the analysis of plasma bubble induced scintillations. One dimensional ionospheric irregularity scale lengths extending from a few hundred kilometres $(147 \mathrm{~km})$ to a few tens of metres $(33 \mathrm{~m})$ are obtained here. However, very interesting results are obtained by treating the PBI scintillations as an autoregressive process and parametrizing the scintillation spectra with a specific AR order and a level of detectability of the process. Three distinct scintillation patterns, each possessing its own characteristic spectral signature, are identified. The onset of scintillations are characterized by isolated fading patterns followed by very rapid quasiperiodic fading patterns due to discrete plasma structures close to the bubble walls. These rapid fluctuations display dominant spectral peak(s) and are resolved using a higher order autoregression and possess lower detectability levels. The well-developed stage of the plasma bubble gives rise to intense scintillations with the S4-index ranging between 0.5-0.9. The corresponding spectra are flat up to high temporal frequencies $(\sim 3 \mathrm{~Hz})$ and hence the spectra corresponds to almost a whitenoise process (close to saturation). These are resolved with a 2nd order filter and have higher detectability levels. Finally, there are a set of spectra with a variety of signatures all attributable to the continuous evolution of plasma process in the ionosphere over short time scales. These can be parametrized with the filter orders from 3 to 6 and detectability levels of $2 \mathrm{~dB}$ to $-3 \mathrm{~dB}$. The filter orders indicate a level of coherence in the process. The higher the order, the greater is the coherence and hence the process is better resolved. Some of these inferences are nearly impossible with conventional spectral techniques like FFT due to their poor resolution. Thus, a new way of looking at plasma bubbles through scintillation effects is described here.

\section{Appendix}

The AR scheme of spectral analysis involves computing the Fourier transform of the model autocorrelation function (ACF) obtained through the AR scheme. A detailed description of this spectral analysis method has already been given by Ulrych and Bishop (1975). Here, some details of (1) evaluating correlation period and optimum data length, (2) evaluation of detectability and frequency resolution and (3) incoherent averaging are given.

\section{Evaluation of the correlation period and optimum data length}

The variance of a time-averaged series is given by

$\sigma_{T}^{2}=\sigma^{2} \frac{T_{0}}{T}$

where $\sigma^{2}$ is the variance of the original sequence, and $T_{0}$ and $T$ are the correlation and averaging periods (in units of sampling interval $\delta t$ ). The averaging period, with a suitable definition, as described later, becomes the optimum extent of the data length. The significance of $T_{0}$ is that the individual data points spaced $T_{0}$ apart may be treated as uncorrelated. Adjacent samples are independent of one another at $T_{0}=1$. The correlation period for a weakly stationary random process, with its mean being constant and ACF dependent on temporal $\operatorname{lag} \tau$, is as given by Popoulis (1965),

$T_{0}=2 \int_{0}^{T}[1-(\tau / T)] \rho(\tau) d \tau$

where $\rho(\tau)$ is the ACF at a lag $\tau$. For instance, for a random process given by exp $\left(-\tau^{2} / \tau_{o}^{2}\right), T_{0}$ from expression (A2) is obtained as $\approx \sqrt{\pi} \tau_{0} / T ; \tau_{0}$ being a suitable decorrelation width. Similarly, correlation period $\left(T_{0}\right)$ for a Markov process, describing a "red noise" spectrum, may be obtained at different averaging periods $(T)$ as shown by Jones (1975). In general, putting in $\rho_{k}$ for an AR process of any order (k), $T_{0}$ may be obtained at different values of $T$ from expression (A2).

Starting from a value of $T=0, T_{0}$ monotonically increases/decreases with $T$ for a Markov (higher-order) AR process. The value of $T_{0}$ is inferred from a "sufficiently" large (and thus optimal) value of data length $T$.

A computer programme generates $T_{0}$ versus $T$ plots, enabling by easy visual inspection the determination of both $T_{0}$ and $T$.

\section{Detectability and spectral resolution of an observed spectrum}

Consider a time series of a non-random process, which is sampled at an interval $\delta t$ and has correlation period $T_{0}$ and optimal data length $T$. The reciprocal of $T_{0}$ represents the (single sided) bandwidth (BW) occupancy of the process. The process is completely resolved at $T_{0}=2 \delta t$, when it is optimally sampled at the Nyquist frequency $(1 / 2 \delta t)$. It also defines the maximum value of one for the detectability $(D)$, defined as

$D \equiv \frac{T_{0}}{2 \delta t}$ 
Thus, as $T_{0}$ becomes smaller than $2 \delta t$ (BW occupancy $>$ Nyquist frequency), detectability $D$ becomes smaller. When $T_{0}$ becomes larger than $2 \delta t$ (BW occupancy < Nyquist frequency), detectability $D$ becomes larger. (Under- and over-sampling in a time series of random data result in aliasing and correlated data points, respectively.) In any case, an "incoherent" averaging over $I_{s}=\left(T / T_{0}\right)^{1 / 2}$ (number of observed spectra) may be computed to obtain an optimally smoothed spectrum Woodman (1985). Alternately, an observed spectrum (obtained from a data of length $I_{s} \times T$ ) may be averaged over $I_{s}$ number of frequencies to obtain an equally smoothed spectrum. The effective resolution bandwidth of the smoothed spectrum would be $\delta f=1 / T$. Spectra of ionospheric scintillations are represented by a detectability $D$ (in $\mathrm{dB}$ ) and have a frequency resolution $\delta f$.

Acknowledgements. This work was carried out at the National Physical Laboratory, India, as a part of a national project called AICPITS', the acronym for All India Coordinated Project of Ionospheric Thermospheric Studies, supported by the Department of Science and Technology. The authors acknowledge the support and encouragement provided by Dr. K.K. Mahajan and Dr. T.R. Tyagi during the course of the work. The authors sincerely thank the anonymous referees for their helpful suggestions and comments.

Topical Editor D. Alcayde' thanks I.S. Batista and another referee for their help in evaluating this paper.

\section{References}

Anderson, D. N., and J. A. Klobuchar, Modeling the total electron content observations above Ascension Island, J. Geophys. Res., 88, 8020-8024, 1983.

Basu, S., S. Basu, J. P. McClure, W. B. Hanson, and H. E. Whitney, High resolution Topside in situ data of electron densities and VHF/GHz scintillations in the equatorial region, J. Geophys. Res., 88, (A1), 403-415, 1983.

Chandra, S., G. D. Vyas, D. R. K. Rao, B. M. Pathan, A. Iype, B. Ram Sekaran, A. Naidu, S. M. Sadique, C. S. Salgoankar, T. R. Tyagi, P. N. Vijayakumar, Lakha Singh, K. N. Iyer, K. N. Pathak, A. K. Gwal, Sushil Kumar, R. P. Singh, U. P. Singh, Birbal Singh, V. K. Jain, G. N. Navneeth, P. V. Koparkar, P. V. S. Rama Rao, P. T. Jayachandran, P. Sriram, N. Y. S. Santa Rao, A. DasGupta, K. Basu, and R. G. Rastogi, Co-ordinated multistation VHF scintillation observations in India during March-April 1991, Ind. J. Radio Space Phys., 22, 69-81, 1993.

Chaturvedi, P. K., and S. L. Ossakow, Nonlinear theory of collisional Rayleigh-Taylor instability in equatorial spread-F, Geophys. Res. Lett., 4, 558-562, 1977.
Crain, C. M., H. G. Booker, and J. A. Ferquson, Use of refractive scattering to explain SHF scintillations, Radio Sci., 14, 125-134, 1979.

Dabas, R. S., and B. M. Reddy, Equatorial plasma bubble rise velocities in the Indian sector determined from multistation observations, Radio Sci., 25, 125-132, 1990.

Franke, S. J., C. H. Liu, and J. P. McClure, Interpretation and modeling of quasiperiodic diffraction patterns observed in equatorial VHF scintillation due to plasma bubbles, $J$. Geophys. Res., 89, A12, 10891-10902, 1984.

Franke, S. J., and C. H. Liu, Analysis of a long period fading component in saturated VHF amplitude scintillation observed at Ascension Island, J. Atmos. Terr. Phys., 49, 421-431, 1987.

Garg, S. C., Y. V. Somayajulu, Lakha Singh, and T. R. Tyagi, Evidence of the development and decay of a postsunset equatorial anomaly at low latitudes, in Proc International Symposium on "Beacon Satellite studies of the earth environment", held at National Physical Laboratory, New Delhi, India, 359-373, 1983.

Heron, M. L., Diffraction from discrete and homogeneously structured ionospheric irregularities, Radio Sci., 14, 97-102, 1979.

Jones, R. H., Estimating variance of time averages, J. Appl. Meteorol., 14, 159-163, 1975.

Kendall, M. G., and A. Stuart, The advanced theory or statistics, vol. 3, pp. 419, 427, 477, Charles Griffin, London, 1966.

Liu, C. H., and S. J. Franke, Experimental and theoretical studies of ionospheric irregularities using scintillation techniques, Radio Sci., 21, 363-374, 1986.

Papoulis, A., Probability, random variables and stochastic processes, (section 9-8), McGraw-Hill, New York, 1965.

Somayajulu, Y. V., S. C. Garg, R. S. Dabas, Lakha Singh, T. R. Tyagi, B. Lokanadham, S. Ramakrishna, and G. Navneeth, Multistation study of nightime scintillations in low latitudes: evidence of control by equatorial $\mathrm{F}$ region irregularities, Radio Sci., 19, 707-718, 1984.

Titheridge, J. E., The diffraction of satellite signals by isolated ionospheric irregularities, J. Atmos. Terr. Phys., 33, 47, 1971.

Trivedi, A. I., M. R. Deshpande, and H. O. Vats Diffraction of ATS-6 signals by ionospheric irregularities - some new aspects, Proc Indian Acad. Sci., 87A, 165-171, 1978.

Ulrych, T. J., and T. N. Bishop, Maximum entropy spectral analysis and autoregressive decomposition, Rev. Geophys., 13, 183-200, 1975.

Vijayakumar, P. N., and T. R. Tyagi, Simulated effects of strong ionospheric scattering on GPS system performance Ind. $J$. Radio Space Phys., 24, 97-101, 1995.

Wernik, A. W., C. H. Liu, and K. C. Yeh, Model computation of radio wave scintillation caused by equatorial ionospheric bubbles, Radio Sci., 15, 559-572, 1980.

Woodman, R. F., Spectral moment estimation in MST radar, Radio Sci., 20, 1185-1195, 1985.

Zalesak, S. T., S. L. Ossakow, and P. K. Chaturvedi, Nonlinear equatorial spread F: The effect of neutral winds and background Pedersen conductivity J. Geophys. Res., 87, (A1), 151166, 1982. 